\title{
HANS-JOCHEN SCHIEWER
}

Die beiden Sankt Johannsen, ein dominikanischer Johannes-Libellus und das literarische Leben im Bodenseeraum um 1300 


\section{Die beiden Sankt Johannsen, ein dominikanischer Johannes- Libellus und das literarische Leben im Bodenseeraum um 1300}

\section{HANS-JOCHEN SCHIEWER}

Für Professor Dr. Kun Ruh

EINE DER berühmtesten deutschsprachigen Handschriften des Mittelalters, der Codex Manesse, bezeugt stellvertretend für andere Handschriften - erhaltene und verlorene - das Interesse an höfischer Literatur in Zürich und im Bodenseeraum um 1300. Diese Handschrift entstand im Laufe des ersten Drittels des 14. Jahrhunderts mit Schwerpunkt am Jahrhundertbeginn. Sie enthält die Lieddichtung von 140 Autoren und bietet zu 138 dieser Liederdichter Autorenbilder. Damit wurde sie zum umfassendsten Sammelbecken mittelhochdeutscher Lyrik von den Anfängen bis in die Zeit der Entstehung der Handschrift selbst hinein. ${ }^{1}$

Ausgangspunkt dieser Sammlung war eine Art 'literarischer Salon', und das bedeutet zu dieser Zeit und auch noch später ein Treffpunkt der politischen und wirtschaftlichen Führungsschicht, dessen Zentrum in diesem Fall die politisch führende Züricher Patrizierfamilie Manesse

\footnotetext{
'Zusammenfassend zuletzt: Codex Manesse, hrsg. von Elmar Mittler und Wilfried Werner, Heidelberg 1988; Die Manessische Liederhandschnift in Zürich, Ausstellungskatalog von Claudia Brinker und Dione Flühler-Kreis, Zürich 1991. Hier finden sich auch Hinweise auf die gleichzeitige Handschriftenproduktion und den Schreibbetrieb in Zürich von Max Schiendorfer (ebda, S.37-40) und Dione FlühlerKreis (ebda, S.41-50). Zur Genese der Handschrift s. Gisela Kornrumpf, 'Die Anfänge der Manessischen Handschrift', in Deutsche Handschriften. 1100-1400. Oxforder Kolloquium 1985, hrsg. von Volker Honeman und Nigel F. Palmer, Tübingen 1988, S.279-296.
} 
bildete. ${ }^{2}$ Ein zeitgenössischer Züricher Dichter, Johannes Hadlaub, stellt uns einige dieser Literaturliebhaber in einem seiner Lieder vor. Sie unterstützen ihn, einen Gunstbeweis seiner Minnedame zu erhalten: ${ }^{3}$

VII

Der vürste von Konstenz, von Zürich diu vürstîn

vil saelig sîn! der vürste ouch sâ

Von Einsidellen, von Toggenburg lobelich

grâf Friderîch, und swer was dâ

Und half alt riet, daz man mich brâchte für sî.

daz tâten hôhe liut, der frume Reginsberger

nach mîner ger ouch was dabî.

VIII

Und der abt von Pêtershûsen tuginde vol

half mir ouch wol. da wâren ouch bî

Edil frowen, hôhe pfaffen, ritter guot.

da wart mîn muot vil sorgen frî.

Ich hâte ir gunst, die doch nit hulfen mir.

her Ruodolf von Landenberg, guot ritter gar,

half mir ouch dar und liebte mich ir.

IX

Dem die besten helfent, daz vervât ouch icht.

diu zuoversicht wart mir wol schîn,

Wan der vürste von Kostenze loblich, gerecht, und her Albrecht, der bruoder sîn,

Und her Rüedge Manesse, die werden man, hulfen mir vür mîn edlen frowen klâr, des manger jâr nie mochte irgân.

\footnotetext{
${ }^{2}$ Als Zeugnis für die literarische Sammelleidenschaft der Manesse kann das Preislied Hadlaubs gelten (SMS 8). Vgl. Max Schiendorfer, Die Schweizer Minnesänger. Bd.1: Text, Tübingen 1990 (=SMS). Indizien sprechen dafür, daß dies nicht der einzige literarische Repräsentationsbereich der Manesse war: Rüdiger soll eine Schwabenspiegel-Handschrift mit panegyrischem Epilog besessen haben (Schiendorfer [Anm.1], S.39).

${ }^{3}$ Ebda, SMS 2.
} 
Die Namenliste liest sich wie ein 'Who's who' der politischen Führungsschicht des Züricher und Konstanzer Raums: ${ }^{4}$

- Heinrich II. von Klingenberg (urkdl. erstmals 1254, gest. 1306), der Bischof von Konstanz, ist die dominierende politische Persönlichkeit des Raums - geschickter Machtpolitiker und Diplomat der Habsburger.

- Er steht in engem Kontakt mit Elisabeth von Wetzikon, der Âbtissin des hochadligen Züricher Fraumünsterstifts (1270-1298), die die geistliche Führungsposition in Zürich inne hat.

- Heinrich von Güttingen ist von 1288 bis 1299 Abt von Einsiedeln; das Kloster besitzt ein Amtshaus in Zürich; die Manesse sind Lehensträger der Einsiedler Benediktiner.

- Der Graf von Toggenburg (Friedrich III. [gest. 1303/05] oder IV. [gest. 1315]) urkundet zusammen mit dem Bischof von Konstanz, der Sohn Friedrichs III., Kraft III. (gest. 1339), wird Chorherr in Konstanz und Probst am Züricher Großmünster.

- Die Freiherren von Regensberg haben am Ende des 13. Jahrhunderts enge Beziehungen zu den Züricher Franziskanern: Ulrich I. wird dort 1281 begraben; Lütold VII. (gest. ca. 1320) urkundet ausschließlich bei den Barfüßern und im Hause des Rüdiger Manesse.

- Dietrich von Kastel, Cousin des Bischofs Heinrich von Klingenberg, ist Abt des Benediktinerklosters Petershausen (1293-1319).

- Der Ritter Rudolf von Landenberg (gest. 1315) gehörte zum engeren Beraterkreis Heinrichs von Klingenberg.

- Und nicht zuletzt wird Rüdiger Manesse selbst genannt: Seine Familie

4 Erstmals ausführlich beschrieben bei Herta Elisabeth Renk, Der Manessekreis, seine Dichter und die Manessische Liederhandschnift, Stuttgart u.a. 1974. Zur Kritik daran s. Max Schiendorfer, 'Ein regionalpolitisches Zeugnis bei Johannes Hadlaub (SMS 2). Überlegungen zur historischen Realität des sogenannten "Manessekreises"', DVjS 112 (1993), 37-65. Schiendorfer bietet wesentliche sachliche Korrekturen zu Renk und situiert das Lied SMS 2 in die einmalige historische Situation des Jahres 1293/94, in der die Stadt Zürich unter der Führung Rüdiger Manesses bemüht war, zu einem versöhnlichen Ausgleich mit den Habsburgern und ihren Anhängern zu kommen. Dieser tagespolitische Aspekt von Hadlaubs Lied, insbesondere dokumentiert in der letzten, etwas isolierten Strophe (SMS 2, XIII), der Preisstrophe auf Bf. Heinrich II. von Klingenberg, reicht aber nicht aus, um den genannten Personen abzusprechen, die 'literarische Szene' zu repräsentieren. Politik, Literatur und Fest bilden einen symbiotischen Zusammenhang im mittelalterlichen Kulturbetrieb. Vgl. auch Ursula Peters, Literatur und Stadt, Tübingen 1983 (Studien und Texte zur Sozialgeschichte der Literatur 7), S.102-104. Zu den folgenden biographischen Angaben neben der genannten Literatur Martin Lassner/Claudia Brinker, 'Pfaffen, Ritter, Bürger', in Die Manessische Liederhandschrift (Anm. 1), S.23-33. 
dominierte um 1300 mit vier weiteren Geschlechtern (Bilgeri, Krieg, Schafli, Mülner) die politische Szene in Zürich. Zum landsässigen Adel bestanden Heiratsbeziehungen (Rüdiger mit der Freifrau Elisabeth von Rüssegg). ${ }^{5}$

Treffpunkt dieser Gesellschaft war sicherlich einer der steinernen Züricher Wohntürme, möglicherweise Münstergasse 22/Napfgasse 2-4 'Zum Großen Erker', der in der Mitte des 13. Jahrhunderts entstand und 1310 urkundlich als 'Manessehof' erwähnt wird. ${ }^{6}$

Weltlicher und geistlicher Adel, geistliches und weltliches Patriziat rekrutieren sich aus ein und denselben Geschlechtern und gehören gleichermaßen $\mathrm{zu}$ den Liebhabern höfischer und späthöfischer Liebeslyrik.' Die Zusammensetzung des 'literarischen Salons' spiegelt sich, wenn auch weniger deutlich, in den Autoren des Codex Manesse: $\mathrm{Zu}$ ihnen gehören Weltkleriker wie Rost von Sarnen mit seinen Liebesliedern, aber auch ein Angehöriger der neuen Orden. Die Mendikanten werden im Preislied Hadlaubs nicht ausdrücklich genannt, stellen aber den einzigen ordensgeistlichen Autor des Codex Manesse: Es handelt sich um den Dominikaner Eberhard von Sax (urkdl. 1309). ${ }^{8}$ Das Autorbild (Abb. 1) ist dem Dedikationstypus verpflichtet und zeigt den 'bredier Eberhart' kniend vor einer Madonna mit Kind. Der im folgenden überlieferte Marienpreis wird im bildeigenen Spruchband der Jungfrau dargebracht: 'Dirre kranke presant. vrowe si dir gesant. emphahe in von mir für gât. dur dinen tugentlichen mút. iemer si von dir

\footnotetext{
${ }^{5}$ Ebda, S.33.

${ }^{6} \mathrm{~J} . \mathrm{E}$. Schneider, 'Die Bauliche Entwicklung', in Die Manessische Liederhandschrift (Anm.1), S.3-19, hier S.19.

${ }^{7}$ Aus dem Geschlecht der Manesse stammten am Ende des 13. Jahrhunderts vier Chorherren am Großmünster und vier Ratsherren, vgl. Lassner/Brinker, 'Pfaffen' (Anm. 4), S.33.

${ }^{8}$ Claudia Brinker, 'Eberhard von Sax', in Die Manessische Liederhandschrift (Anm. 1), S.119-129; vgl. Renk, Manessekreis (Anm. 4), S.33-35. Bei Heinrich von Mure ist die Ordenszugehörigkeit nicht geklärt; Dominikaner ist denkbar.
} 
Abb. 1 Große Heidelberger Liederhandschrift/Codex Manesse. Heidelberg, UB, cpg 848 , fol. $48^{v}$.

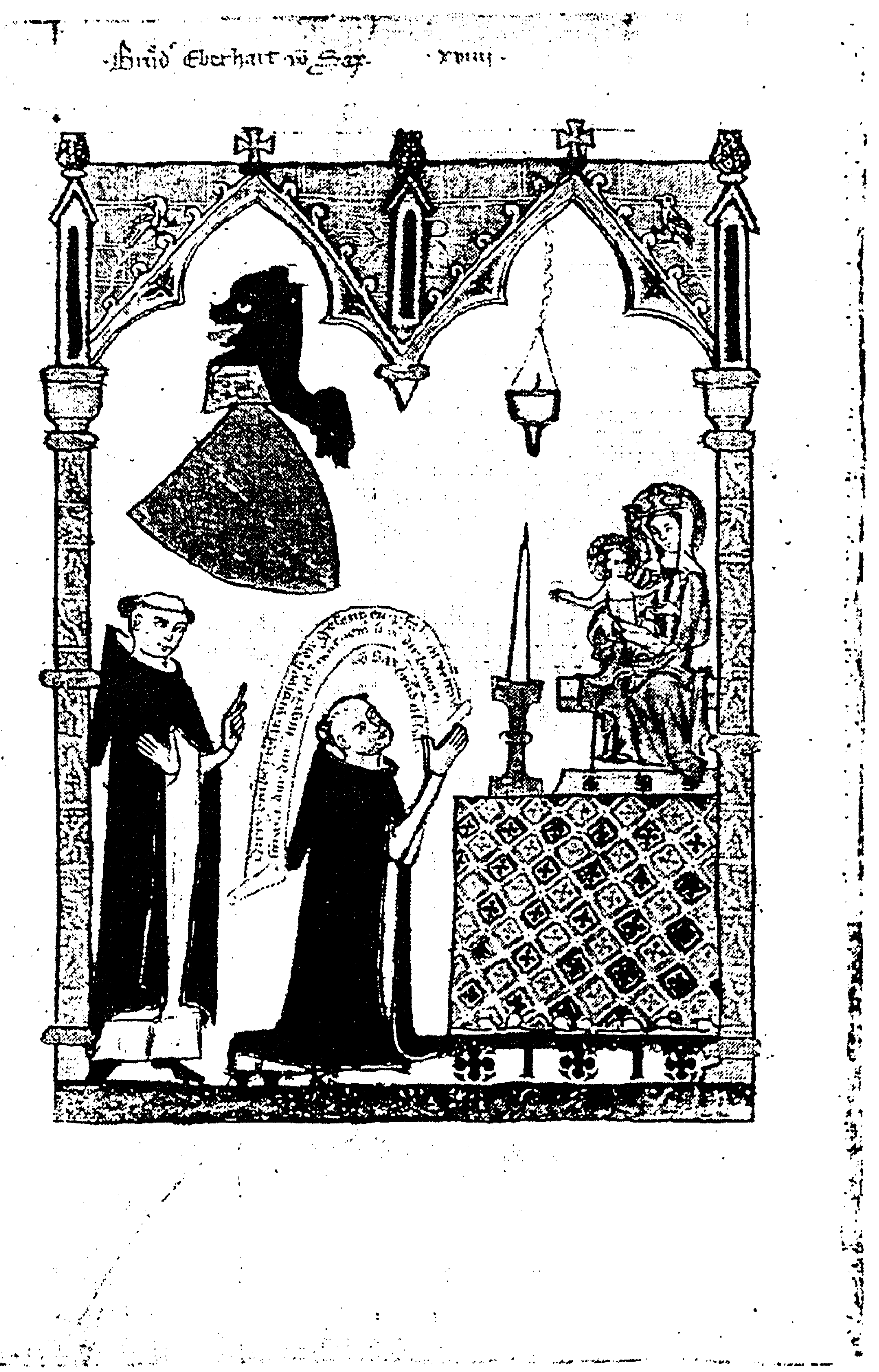


bewart. von Sax bruder Eberhart'. ${ }^{9}$ Der Autor entstammt demselben Ostschweizer Adelsgeschlecht wie der Minnesänger Heinrich II. von Sax (urkdl. 1253, 1257, 1258) und gehörte nach Lehrjahren im Konstanzer Nikolauskloster zum Züricher Predigerkloster. ${ }^{10}$ Damit ist ein Brückenschlag vom Codex Manesse und seinen Auftraggebern zu den Dominikanern möglich. Das Auftreten eines zeitgenössischen dominikanischen Autors in dieser Handschrift erweckt nun Hoffnungen darauf, daß es zu dieser Zeit schon ein entwickeltes deutschsprachiges dominikanisches Schrifttum gegeben haben könnte, zumal dem Orden die Betreuung der Frauenkonvente oblag und die Franziskaner schon im ganzen 13. Jahrhundert deutliche Spuren ihrer literarischen Tätigkeit in der Volkssprache hinterlassen hatten, und zwar gerade im Hinblick auf die cura der weiblichen Religiosen und der Klarissen. ${ }^{11}$ Ein Blick in die Literaturgeschichten enttäuscht diese Erwartung jedoch: ${ }^{12}$ Dominikanisches Schrifttum in deutscher Sprache beginnt erst während der Entstehung des Codex Manesse. Meister Eckhart ist der erste prominente Vertreter mit umfangreichem Oeuvre, dessen Entstehungszeit - soweit es die cura frommer Frauen betrifft - nicht vor 1314 anzusetzen sein dürfte. ${ }^{13}$ Aus der gleichen Zeit stammt die Hand-

\footnotetext{
${ }^{9}$ Text nach der Handschrift, Abbreviaturen sind aufgelöst. Auf den singulären Charakter dieses Bildes im Rahmen der Handschrift kann hier nicht eingegangen werden. Vgl. Martina Wehrli-Johns, Geschichte des Zürcher Predigerkonvents (12301524). Mendikantentum zwischen Kirche, Adel und Stadt, Zürich 1980, S.202.

${ }^{10}$ Wehrli-Johns, Zürcher Prediger (Anm. 9), S.244.

"Kurt Ruh, 'David von Augsburg und die Entstehung eines franziskanischen Schrifttums in deutscher Sprache' (1955), in K.R., Kleine Schriften, Berlin/New York 1984, II, 46-67. Eine Neubewertung der handschriftlichen Überlieferung bei Karin Schneider, Gotische Schriften in deutscher Sprache. I. Vom späten 12. Jahrhundert bis um 1300, Wiesbaden 1987, S.253-256.
}

${ }^{12}$ Eine diffuse Verbindung im Rückgriff auf den 'so schwer faßbare[n] Geist der Zeit' stellt Renk (Manessekreis [Anm. 4], S.29) her, ohne über eine geeignete Textbasis zu verfügen. Erwăhnung finden nur Hugo Ripelin OP (gest. 1270) und die Schwesternviten. Mehr Profil gewinnt das Bild bei Wehrli-Johns (Zürcher Prediger [Anm. 9], S.203-205), die mit dem um 1320 wirksamen Dominikaner Marchwart Biberli einen weiteren Anknüpfungspunkt hat.

${ }^{13}$ Kurt Ruh, Meister Eckhart. Theologe, Prediger, Mystiker, 2. überarb. Aufl., München 1989, S.18-46, 60-71, 95-114. Trotz aller Schwierigkeiten mit der Chronologie Eckhartscher Werke (z.B. ebda, S.29f.) darf wohl als sicher gelten, daß nur die Reden der Untenveisung (ebda, S.31) und die Predigten des Paradisus anime 
schrift des Solothumer oder Marchwart Biberli-Legendars, das aufgrund seiner Schlußbemerkung zweifelsfrei für Dominikanerinnen bestimmt war und als dessen Redaktor der Züricher Dominikanerlesemeister Marchwart Biberli (urkdl. 1320-1326) gilt; zugleich wird Marchwart mit der frühesten deutschen Bibelübersetzung in Verbindung gebracht. ${ }^{14}$ Mithin beginnt deutschsprachiges dominikanisches Schrifttum in nennenswertem Umfang erst im frühen 14. Jahrhundert.

Eine Reihe von Johannespredigten - anfangs auf den Evangelisten, später auf den Baptisten - und weitere Texte in einer Foliohandschrift des 15. Jahrhunderts ändern nun diesen Befund. Der Codex stammt aus dem bairischen Raum und gehört heute zur Gräflich Schönbornschen Bibliothek in Pommersfelden in der Nähe von Bamberg. ${ }^{15}$ Der erste Teil der Handschrift enthält eine Apokalypse, die 'Zeichen des Johannes', eine Legende auf Johannes Evangelista, eine Predigt auf Jakobus den Älteren, vier Predigten auf den Evangelisten, eine Predigt auf Agnes und drei Predigten auf Johannes den Täufer. Neben Bernhard von Clairvaux und Vinzenz von Beauvais werden vier weitere Autoren genannt, die mit einer Ausnahme bisher unbekannt sind. Es handelt sich in der Reihenfolge ihres Auftretens um:

intelligentis Produkte seines Erfurter Priorats und seines Vikariats in der Saxonia sind. Die Mehrzahl seiner deutschen Predigten, d.h. der Predigten, die im Rahmen der cura monialium entstanden, gehören in die Zeit seines Generalvikariats (seit dem Ende des zweiten Pariser Magisteriums im Sommer 1313).

14 Wehrli-Johns, Zürcher Prediger (Anm. 9), S.203-205; Marianne Wallach-Faller, Ein alemannischer Psalter aus dem 14. Jahrhundert. Hs. A.IV.44 der Universitätsbibliothek Baseh, BL 61-178, Freiburg i.Ü. 1981 (Spicilegium Friburgense 27), S.100-109; dies.: 'Die erste deutsche Bibel? Zur Bibelübersetzung des Zürcher Dominikaners Marchwart Biberli', ZfdA 110 (1981), 35-57; Karl-Ernst Geith, 'Marchwart Biberli und das Solothurner Legendar Cod. S.451', ZfdA 111 (1982), 921; Marianne Wallach-Faller, 'Ein mittelhochdeutsches Dominikanerinnen-Legendar des 14. Jahrhuderts als mystagogischer Text?', in Abendländische Mystik im Mittelaler. Symposion Kloster Engelberg 1984, hrsg. von Kurt Ruh, Stuttgart 1986 (Germanistische Symposien-Berichtsbände 7), S.388-401. Zusammenfassend zuletzt Werner Williams-Krapp, Die deutschen und niederländischen Legendare des Mittelalters, Tübingen 1986 (TTG 20), S.24f.

${ }^{15}$ Pommersfelden, Gräflich Schönbornsche Bibliothek, Ms. 120 (olim 2868). Vgl. Tilo Brandis, Handbuch der Handschriftenbestände in der Bundesrepublik Deutschland, I, Wiesbaden 1992, S.412. 
- Bruder conrat von liebenberg der lesenmaister zu ze (!) constancze was $\left(61^{\text {vb }}\right)$,

- Bruder hainrich von schaffhausen der arler der lesenmaister zu zurich was $\left(79^{\text {ra }}\right)$,

- vnser liber vater bruder rudolff von clingenberg $\left(89^{\text {ra }}\right)$,

- vnser lieber vater bruder huch der prouincial $\left(103^{\text {rb }}\right)$.

Die Nennung der Orte Konstanz, Schaffhausen und Zürich verweist auf den Bodenseeraum, die Funktionsbezeichnung Lektor oder Provinzial auf die Bettelorden. 'Bruder huch der prouincial' kann mit Hugo von Konstanz O.P. identifiziert werden, der von 1300-1304 Provinzial der Teutonia war.

Wenn es nun gelingt, die vier Autoren historisch nachzuweisen und als Gruppe dominikanischer Autoren im Bodenseeraum um bzw. vor 1300 zu sichern, wäre es erstmals möglich, dominikanische Literatur in größerem Umfang in der Generation vor Meister Eckhart im oberdeutschen Raum nachzuweisen. Gleichzeitig erhalten wir damit Einblick in die frühe cura monialium der Dominikaner, denn für diesen Bereich dürften die Texte bestimmt gewesen sein.

Ziel der folgenden Untersuchung ist es nun,

- anhand der Autornennungen Entstehungszeit, -ort und -kontext der Predigten auf Johannes Baptista und Evangelista sowie Jakobus und Agnes zu erschließen und zu sichern,

- die Abfolge der Texte auf eine planvolle Anlage hin zu befragen,

- eine Predigt auf den Evangelisten exemplarisch vorzustellen und mit älteren Johannespredigten zu kontrastieren,

- die Stellung der Predigten im Rahmen des dominikanischen Schrifttums zu bestimmen

- und ihren Platz in der literarischen Landschaft am Bodensee um 1300 zu skizzieren. ${ }^{16}$

\footnotetext{
${ }^{16}$ Im Rahmen der hier gegebenen Möglichkeiten kann nur ein erster Überblick zu den Autoren und ihren Texten geboten und der literarische sowie kunsthistorische Horizont nur knapp beschrieben werden. Eine erschöpfende prosopographische und quellenkritische Studie sowie die Klärung weiterer offener Fragen bleiben der geplanten Edition der Handschrift vorbehalten.
} 


\section{Die Autoren}

\section{'Huch der prouincial'}

'Huch' bzw. Hugo wurde schon kurz vorgestellt, soll hier aber nochmals etwas ausführlicher bekannt gemacht werden. Martina Wehrli-Johns schrieb die Geschichte des Züricher Predigerkonvents (1230-1524) und hat die historischen Daten zu Hugo zusammengetragen; seine literarische Tätigkeit blieb ihr allerdings verborgen: ${ }^{17}$ Sein persönliches Siegel trägt die Umschrift 'de Scaphusa'. Er stammt also aus dem stadtadligen Geschlecht 'der Schaffhausen', das in Konstanz ansässig war. Von 1279. 1288 war er Lektor im Konstanzer Predigerkloster St. Nikolaus und von 1288-1295 Prior der Züricher Dominikaner. Er wurde dann Vikar des Provinzialpriors und anschließend noch einmal Prior der Züricher Dominikaner. Auf dem Generalkapitel in Löwen 1300 wählte man ihn zum Provinzial der Teutonia. Drei Jahre später ist er gestorben: 'Bruder Hugo von Zürich, gar ein seliger andechtiger man und waz by III jaren an dem ampt und starb daran'. ${ }^{18}$

In seinen verschiedenen Funktionen trägt er die Namen Hugo von Schaffhausen, Hugo von Konstanz und Hugo von Zürich. Er machte eine steile Ordenskarriere vom Lektor in Konstanz zum Prior in Zürich, Vertreter des Provinzials und Provinzial selbst. Wichtig für uns ist seine Zusammenarbeit mit Hermann von Minden (1286-1290), der mit seinen admonitiones und Instruktionen wesentlichen Anteil an einer Neuorganisation und Intensivierung der cura monialium hatte. ${ }^{19}$ Die lange Zeit umstrittene cura für fromme Frauen wurde auf dem Generalkapitel 1257 in Florenz endgültig akzeptiert und in einer Bulle Clemens IV. vom 6. Februar 1267 nochmals ausdrücklich als Pflicht dem

17 Wehrli-Johns, Zürcher Prediger (Anm. 9), S.50, 175-179; zur literarischen Tătigkeit Hugos Dagmar Ladisch-Grube/Kurt Ruh, in ${ }^{2}$ VL IV (1983), 232f. Walter Muschg (Die Mystik in der Schweiz 1200-1500, Frauenfeld/Leipzig 1935) identifiziert Hugo von Konstanz ohne weitere Begründung mit dem in den Katharinentaler Nonnenviten genannten Konstanzer Lesemeister Hugo von Staufenberg (S.193).

${ }^{18}$ P. von Loe, Statistisches über die Ordensprovinz Teutonia, Leipzig 1907 (Quellen und Forschungen zur Geschichte des Dominikanerordens in Deutschland 1), S.27.

${ }^{19}$ Gabriel Löhr, 'Drei Briefe Hermans von Minden O.P. über die Seelsorge und die Leitung der deutschen Dominikanerinnenklöster', Römische Quartalschrift 33 (1925), 159-167. 
Orden auferlegt. ${ }^{20}$ Daß Hugo sich dieser Pflicht nicht entzog, zeigt seine Tätigkeit als confessor bei den Ötenbacher Dominikanerinnen in Zürich und in Töss bei Winterthur. Ein Reflex dieser Funktion ist die Bezeichnung vater (103ra) in unserer Handschrift. Sie weist ihn als pater confessarius aus. Seine ausdrückliche Aufgabe war also die Seelsorge, die cura animarum. Darüber hinaus wird er in der Handschrift 'der prouincial' genannt, so daß die erstmalige Niederschrift seiner Predigt auf Johannes Baptista in der vorliegenden Form nicht vor 1300 erfolgt sein konnte.

Bestehende Zweifel am Alter dieser Predigt räumt eine Parallelüberlieferung in einer alemannischen Handschrift des 14. Jahrhunderts aus, auf die Ruh schon hinwies. ${ }^{21}$ Zweifel an der literarischen Tätigkeit Hugos von Konstanz zerstreut eine Handschrift aus dem späten 13. Jahrhundert, die die Größe einer Streichholzschachtel hat (Karlsruhe, LB, St. Georg. perg. 31). Sie überliefert je eine weitere Predigt auf Johannes Evangelista und Baptista unter der Überschrift: 'Dise predie tet vns Brvder Hugo der lesemaister von Constence von dem lieben v̄ dem guten sant Johannes ewangeliste'. ${ }^{22}$ Lektor in Konstanz war Hugo von 1279-1288, damit erhalten wir die Bestätigung, daß seine literarische Tätigkeit in der cura kein Einzelfall ist, sich über mehr als 20 Jahre erstreckte und wohl eine seiner wesentlichen Aufgaben war.

\section{Rudolf von Klingenberg}

Die Herkunftsbezeichnungen der weiteren Autoren unserer Handschrift Liebenberg, Schaffhausen und Klingenberg verweisen in denselben

20 John Freed, "Urban Development and the "cura monialium" in ThirteenthCentury Germany', Viator 3 (1972), 311-327; Otto Langer, Mystische Erfahnung und spirinuelle Theologie. $Z u$ Meister Eckharts Auseinandersetzung mit der Frauenfrömmigkeit seiner Zeit, München 1987 (MTU 91), S.9-23.

${ }^{21}$ Ruh (Anm. 17), Sp.233; Königsberg, UB, cod. 898, 97'-101, vgl. dazu Emil Steffenhagen, 'Die altdeutschen Handschriften zu Königsberg', ZfdA 13 (1867), 501574, hier 509f.; Siegmund Prillwitz, Überliefenungsstudie zum 'Barlaam und Josaphat' des Rudolf von Ems. Eine textkritisch-stemmatologische Untersuchung, Kopenhagen 1975, S.53.

2 Alfred Holder, 'Zwei Predigten des Lesemeisters Hugo von Constanz', ZfdPh 9 (1878), 29-43, hier 29. 
geographischen Raum. Ihre Identifizierung ist jedoch ungleich schwerer, da die Quellenbasis für prosopographische Studien nicht gerade günstig ist und insbesondere für das Konstanzer Predigerkloster geradezu katastrophal genannt werden muß, da im 19. Jahrhundert Bibliothek und Archiv des Klosters auf ungeklärte Weise verschwanden. ${ }^{23}$

Beginnen wir mit dem klangvollsten und bedeutendsten $\mathrm{Na}$ mensträger Rudolf von Klingenberg. Die Klingenberger gehörten zum Kreis der literarisch Interessierten, genannt im Umkreis des Codex Manesse, vertreten insbesondere durch den anfangs schon vorgestellten Bischof von Konstanz, Heinrich II. von Klingenberg, und seinen Bruder Albrecht. Die Nachbarschaft zu Dominikanern aus Konstanz und Zürich erlaubt es nun, Rudolf als einen Angehörigen dieses Thurgauischen Ministerialengeschlechts zu identifizieren. Er ist urkundlich als Angehöriger des Konstanzer Predigerklosters nachweisbar: 1279 gilt seine Zeugenschaft einer Rente für die samenunge der Schwestern am Brül in St. Gallen (seit 1371 Dominikanerinnenkloster St. Katharina) ${ }^{24}$ Er wird zusammen mit dem Prior genannt: 'Acta sunt hec apud sanctum Gallum in curia nostra, presentibus fratre Cvnrad priore et fratre Rvdolfo de Clinginberg ordinis Predicatorum de Constantia'. ${ }^{25} 1291$ tritt er zusammen mit dem Offizial von Konstanz, dem höchsten Vertreter der bischöflichen Gerichtsbarkeit, bei einem Verkauf von Liegenschaften auf ('in presentia fratris Rudolfo de Clingenberch ... ordinis fratrum predicatorum'). ${ }^{26}$

Eine Einordnung Rudolfs in den Stammbaum der Klingenberger ist nicht unproblematisch: Identität Rudolfs mit Rudolf I. von Klingenberg, der von 1236-1273 als miles urkundet, ist möglich, aber unwahrscheinlich. Vermutlich handelt es sich um einen Sohn Rudolfs I.

${ }^{23}$ M. Brigitta Hilberling OP, Das Dominikanerkloster S. Nikolaus auf der Insel vor Konstanz. Geschichte und Bedeutung, Sigmaringen/München 1969.

${ }^{24}$ Th.K. Vogler OP, Geschichte des Dominikanerinnen-KIosters St. Katharina in St. Gallen 1228-1607, Freiburg i.Ü 1938.

${ }^{25}$ Chartularium Sangallense, Bd. 4 (1266-1299), bearb. von O.P. Clavadetscher, Sigmaringen/St. Gallen 1985, Nr. 2031 zum 7. Juni 1279.

${ }^{20}$ Urkundenbuch der Stadt und Landschaft Zürich, Bd. 6, bearb. von J. Escher und P. Schweizer, Zürich 1903, Nr. 2122. 
In diesem Fall wäre Rudolf ein Vetter Heinrichs II. von Klingenberg, der von 1293-1306 den Konstanzer Bischofsstuhl innehatte. ${ }^{27}$

In Rudolf von Klingenberg treffen wir also auf einen Dominikaner, der über seine Familienzugehörigkeit unmittelbar mit dem Literaturkreis in Zürich und dem Konstanzer Bischofshof verbunden gewesen war. Die Beziehungen zu seinem mutmaßlichen Vetter, dem Konstanzer Bischof, können nicht schlecht gewesen sein, denn er urkundet zusammen mit dem bischöflichen Offizial. Ein weiterer Hinweis auf das gute Einvernehmen zwischen Bischof und Bettelorden ist die Tatsache, daß Heinrich von Klingenberg in seinem Testament u.a. die Dominikaner berücksichtigt. ${ }^{28}$

Denkbar ist, daß Rudolf nicht der einzige literarisch tätige Prediger aus dem Hause der Klingenberger war. 'bruder Johans von Clingenberg, ain brediger' taucht 1299 in einer Konstanzer Urkunde auf und ist dann 1314 als Prior der Konstanzer Dominikaner belegt. ${ }^{29}$ Zwei Predigten eines gleichnamigen Dominikaners werden aus dem Nürnberger Katharinenkloster überliefert, d.h. aus dem 15. Jahrhundert und damit gleichzeitig zu den Texten unserer Handschrift. Inhaltlich gibt es keine Gründe, eine frühe Entstehung auszuschließen, zumal das Gleichnis vom Goldenen Berg des Königs von Frankreich bei Nikolaus von Straßburg (urkdl. 1318-1331) ebenfalls auftaucht. ${ }^{30}$

${ }^{27}$ Johann Jakob Rüeger, Chronik der Stadt und Landschaft Schaffhausen, Bd. 2, Schaffhausen 1892, S.668-683, $1143 f$.

${ }^{28}$ Regesta Episcoporum Constantiensium, Bd. 2, Innsbruck 1905, Nr. 3118.

29 Thurgauisches Urkandenbuch, Bd. 3 (1251-1300), redig. von Friedrich Schaltegger, Frauenfeld 1925, Nr. 959 (Konstanz, 12. Oktober 1299); ebda, Bd. 4 (1300-1340), redig. von Friedrich Schaltegger und Ernst Leisi, Frauenfeld 1931, Nr. 1194. In der letztgenannten Urkunde geht es um die Vergabung einer Immobilie durch den Freiherrn Burkhard von Eschlikon zugunsten der Dominikanerinnen in Katharinental bei Diessenhofen.

${ }^{30} \mathrm{~J}$. Werlin, 'Mystikerzitate aus einer Nürnberger Predigthandschrift', Archiv für Kulturgeschichte 43 (1961), 240-259, hier 252-259. Der Hinweis auf die parallele Verwendung des Gleichnisses bei Kurt Ruh, in ${ }^{2}$ L IV (1983), 659. 
Das Engagement der Konstanzer Dominikaner für die frommen Frauen am Brül in St. Gallen zeigt zudem, daB sich ihre Aufgaben in der cura monialium nicht auf die inkorporierten Frauenkonvente beschränkte, sondern auch den Lebensgemeinschaften von Religiosen galt, die nicht ordensmäBig gebunden waren.

\section{Heinrich von Schaffhausen und Konrad von Liebenberg}

Die beiden verbleibenden Autoren, Heinrich von Schaffhausen und Konrad von Liebenberg, sind bislang nicht sicher urkundlich nachweisbar.

'hainrich von schaffhausen der arler der lesenmaister zu zurich' gehörte vermutlich ebenfalls zu der stadtadligen Konstanzer Familie 'der Schaffhausen', aus der auch Hugo von Konstanz stammt. Der Beiname 'arler' (Pflugmesser) ist in Konstanzer Urkunden bisher nicht nachweisbar. Nur Schaffhauser Urkunden der 1. Hälfte des 13. Jahrhunderts nennen ein solches Geschlecht. ${ }^{31}$ Ohne weitere personengeschichtliche Untersuchungen kann hier nur vermutet werden, $\mathrm{da} B$ das Schaffhauser Geschlecht der Arler um die Mitte des 13. Jahrhunderts nach Konstanz zog und sich dann dort nach ihrem Herkunftsort 'von Schaffhausen' nannte. Identität könnte mit einem 'fratre Hainrich dicto de Constantia ordinis Predicatorum domus Constantiensis' bestehen, denn der oben vorgestellte Hugo nannte sich ebenfalls 'von Konstanz' und 'von Schaffhausen'. Er wird zusammen mit dem Prior Konrad unter den testes einer 1279 ausgestellten Salemer Urkunde genannt. ${ }^{32}$ Als Lektor des Züricher Dominikanerklosters ist er nicht nachweisbar. ${ }^{33}$ Dies kann angesichts der dürftigen Quellenlage nicht erstaunen. Die Anhaltspunkte reichen aber aus, um ihn als einen Zeitgenossen Hugos von Konstanz und Rudolfs von Klingenberg anzusehen.

${ }^{31}$ Urkandenbuch Zürich (Anm. 26), Bd. 3, Zürich 1895f., Nr. 1076 = Schaffhauser Urkundenregister Nr. 143. Der Schultheiss von Schaffhausen verkündet ein Urteil: 'Hii autem presentes fuerunt ... Rudolfus et $\mathrm{H}$. dicti Arlere'.

${ }^{32}$ Chartularium Sangallense (Anm. 25), Nr. 2028.

${ }^{33}$ Wehrli-Johns, Zürcher Prediger (Anm. 9). 
Noch schlechter sieht die Quellenlage fứr Konrad von Liebenberg aus. ${ }^{34}$ Im Kontext der bekannten drei Autoren kann es sich nur um einen Angehörigen des Ministerialengeschlechts der Liebenberger handeln, deren gleichnamige Stammburg in der Grafschaft der Kyburger in der Nähe Winterthurs lag. Im 13. Jahrhundert urkundet ein 'Ulricus' und ein 'Cunradus' als miles, so daß zumindest Konrad als Leitname für dieses Geschlecht gesichert werden kann. Frauen der Liebenberger traten in der 2. Hälfte des 13. Jahrhunderts in das Dominikanerinnenkloster Töss ein. ${ }^{35}$ Urkundliche Nachrichten über die Liebenberger fehlen im 14. Jahrhundert, so daß sie vermutlich um 1300 ausgestorben sein werden. ${ }^{36}$

Konrad von Liebenberg ist nach Auskunft der Handschrift Lektor in Konstanz und Autor der Predigt auf Jakobus den Älteren $\left(61^{\text {vb }}-69^{\text {ra }}\right)$. Innerhalb dieser Predigt werden die Wundertaten des Jakobus und die Pilgerfahrt nach Santiago di Compostella erwähnt. Konrad kommentiert dies mit der Bemerkung, da $\beta$ das Wirken des Heiligen seit nunmehr 1300 Jahren andauere $\left(67^{\mathrm{b}}\right)$. Dieser Hinweis kann uns darin bestärken, ihn als Mitbruder Hugos von Konstanz, Rudolfs von Klingenberg und Heinrichs von Schaffhausen anzusehen.

Fassen wir die prosopographischen Untersuchungen zusammen, ergibt sich folgendes Bild:

- Wir haben es bei den vier Autoren unserer Handschrift mit einer Gruppe von Dominikanern zu tun, die zwischen 1279 und 1304 in Konstanz und Zürich nachweisbar sind bzw. deren Familien in den genannten Städten oder im Nordschweizer Raum ansässig waren.

- Sie stammen alle aus stadt- oder landadligen Familien dieser Region.

- Die beiden als vater bezeichneten Prediger Hugo von Konstanz und Rudolf von Klingenberg können direkt in Verbindung mit der cura monialium gebracht werden - Rudolf locker in seiner Verbindung zur

${ }^{34}$ Volker Honemann, in ${ }^{2} V L \mathrm{~V}(1985), 218$.

${ }^{35}$ Marie-Claire Däniker-Gysin, Geschichte des Dominikanerinnenklosters Tö $\beta$ 1233-1525, Winterthur 1957 (289. Neujahrsblatt der Stadtbibliothek Winterthur), S.98.

${ }^{36}$ Oberbadisches Geschlechterbuch, bearb. von J. Kindler von Knobloch, Bd. 2 , Heidelberg 1905, S.504. 
samenunge frommer Frauen in St. Gallen, Hugo unmittelbar als confessor in Töss bei Winterthur und Ötenbach in Zürich.

- Der einzige bisher bekannte Autor dieser Gruppe war Hugo von Konstanz.

\section{Der Aufbau der Handschrift}

Die Handschrift ${ }^{37}$ beginnt auf den ersten zwanzig Blättern mit der Apokalypse des Johannes, die nach mittelalterlichem Verständnis dem Evangelisten zugeschrieben wird: 'Dis ist das buch das der gut sant Johannes ewangelista gescriben hat von der heimlichen offenwarung, die im got offent' $\left(1^{r a}-20^{\text {rb }}\right) .^{38}$ Der folgende Text, die 'zaichen von sant johannes' genannt, ist dem apostolischen Wirken und den Wundertaten des Johannes gewidmet, wie sie in den Heiligenviten dargestellt werden $\left(20^{\text {th }}-31^{\text {tb }}\right)$; den Abschluß bilden vier posthume Wunderberichte $\left(31^{\text {th }}\right.$ $\left.33^{\text {vb }}\right)$. Es handelt sich dabei um eine Kompilation, deren Quellenbasis bisher nicht bestimmt wurde und $\mathrm{zu}$ der es keine bekannte Parallelüberlieferung gibt. Mit Blatt 33 beginnen die Predigten, die erst dem Evangelisten gewidmet sind und dann dem Baptisten. Das Bindeglied zwischen beiden Predigtgruppen bildet eine Agnes-Predigt ( $9 T^{T^{t}}$ $\left.103^{\text {rb}}\right)$. Eröffnet wird die Reihe der Johannes-Predigten durch eine Autorität mystischer Literatur, Bernhard von Clairvaux $\left(33^{\text {vb }}-48^{\text {rb }}\right)$. Die Zuschreibung ist fraglich. Dies ändert aber nichts an der programmatischen Stellung dieser Predigt am Anfang und der Signalwirkung von Bernhards Namen im Kontext mystischer Frömmigkeit: Bernhard steht mit seinen Hoheliedpredigten für die hochmittelalterliche Ausprägung der Idee einer bräutlichen Vereinigung zwischen der einzelnen Seele und

${ }^{37}$ Kodikologische Daten des ersten Teils (1-131): Papier, $300 \times 215,1 \mathrm{Hd}$., zweispaltig, 34 Zeilen, $1^{\mathrm{x}}$ A-Initiale, rote Lombarden, rubrizier, rote Überschriften, alte Foliierung, Mitte 15. Jh., Provenienz unbekannt, Schönborn-Einband.

38 Die Apokalypse läBt sich keinem der publizierten Übersetzungszweige zuordnen. Vgl. Wilhelm Walther, Die deutschen Bibelübersetzungen des Mittelalters, 3 Bde., Braunschweig 1889-1892 (Nachdruck: Nieuwkoop 1966), Sp.283-286 u. 551f. Damit ist weder gesichert noch ausgeschlossen, daß die Úbersetzung in der 2 . Halfte des 13. Jh. entstand. Insgesamt ist die Forschungssituation zu Zahl, Überlieferung und Gruppierung deutschsprachiger Apokalypsen nicht ausreichend. Vgl. Nikolaus Henkel, 'Eine deutsche Apokalypse des 15. Jahrhunderts. Ein Fundbericht', Vestigia Bibliae 9/10 (1987/88 [1991]), 172-180. 
Gott. ${ }^{39}$ Allerdings ist in dieser Johannes-Predigt die unio mystica kein Thema, es geht nicht um die persönliche Gottesschau und den Weg dorthin, sondern - wenn man so will - um eine historische Liebesbeziehung: diejenige zwischen Christus und Johannes. Die Minnethematik steht somit zwar im Mittelpunkt dieser und der folgenden Predigten, aber es geht nicht um die Gottesminne der frommen Frauen selbst. Eine Ausnahme bildet hier nur die Agnes-Predigt. Die Beziehung Christus Johannes könnte aber als Modell dienen, das - mittelbar - auf eine brautmystische Konstellation gedeutet werden kann. Die mittelalterliche Deutungstradition der Gestalten des Täufers und des Evangelisten macht sie immer wieder zu Vorbildern jungfräulichen Lebens. ${ }^{40} \mathrm{Im}$ Speculum Virginum, entstanden wohl in den vierziger Jahren des 12 . Jahrhunderts, wird der Evangelist mit der sponsa des Hohelieds gleichgesetzt. Ich zitiere die mittelniederländische Übersetzung aus dem 14. Jahrhundert: 'de bruut Christi di bi sent iohan oec beteickent is'. ${ }^{11}$ Explizite Hinweise auf ein solches Verständnis enthalten die Texte allerdings nicht. Mithin handelt es sich trotz bildreicher und minnesatter Sprache nicht um mystische Predigten im strengen Sinne. Ich zitiere zur Illustration aus dem Beginn der Bernhard zugeschriebenen Predigt:

Das ist der aller seligste bott vnd der hohe ewangeliste Der aller heiligste mensch sant Johannes Ein gesell der engel Ein gemynter gotz Ein ander sunn der obersten mägd marien Ein spiegel aller keuschheit Ein cron aller propheten Der oberst aller marterer Ein ere vnd glorie aller beichtiger Ein wirdickeit aller geistlichen Ein bilder aller maid Ein liecht

${ }^{39}$ Kurt Ruh, 'Die Hoheliederklärungen Bernhards von Clairvaux und Wilhelms von St. Thierry', in 'Minnichlichiu gotes erkennusse'. Studien zur frühen abendländischen Mystiktradition, hrsg. von Dietrich Schmidtke, Stuttgart-Bad Cannstatt 1990 (Mystik in Geschichte und Gegenwart Abt.I. Bd. 7), S.16-27.

${ }^{40}$ Matthäus Bernards, Speculum virginum. Geistigkeit und Seelenleben der Frau im Hochmittelalter, Köln/Graz 1955 (Forschungen zur Volkskunde 36/38), S.20, 61-65. Zur Datierung vgl. die editio princeps: Jutta Seyfarth, Specvlvm virginvm, Turnhout 1990 (Corpus Christianorvm Continuatio Mediaeualis 5), S.32*-37*.

${ }^{41}$ Zitat bei Irene Berkenbusch, "Mystik und Askese. "Sankt Trudperter Hohes Lied" und "Speculum Virginum" im Vergleich', in 'Minnichlichiu gotes erkennusse' (Anm. 39), S.43-60, hier S.46. Zur bevorzugten Stellung des Evangelisten in den dominikanischen Klosterchroniken und Schwesternviten vgl. Hieronymus Wilms, Das Beten der Mystikerinnen, Leipzig 1916 (Quellen und Forschungen zur Geschichte des Dominikanerordens in Deutschland 11), S.82f. 
der cristenhait Ein gezierde aller der welt. Ein zederbawm des paradeis Ein gebietter des himels. Ein form aller volkomenheit $\left(33^{\text {vb }}-34^{\text {na }}\right)$.

Diese Flut von Johannes-Metaphern findet eine Entsprechung in der Agnes-Predigt, in deren Mittelpunkt nicht die jungfräuliche Märtyrerin selbst steht, sondern die Vorliebe Christi für die Jungfrauen und damit zugleich der sonst ausgeklammerte Aspekt der unio:

so hat er sich doch hart vngeleich vereiniget mit den megden mit sunderlicher haimlikeit vnd mit ausgenomener zemen fugunge der mahelschaft denn mit chainer creature $\left(98^{\text {ra }}\right)$.

Diese Verherrlichung des Jungfrauenstandes verweist die Predigten unzweifelhaft in die cura monialium. Die Jungfrau wird nun in der verherrlichenden Bildsprache dem Evangelisten gleichgestellt:

ein maget ist ein tochter des vater ein maget ist ein muter des sunes ein maget ist ein tempel des heiligen gaistes ein maget ist ein eigenlich bilde der hohen driualtikait ein maget ist ein schrein der gotlichen minne ein maget ist ein junckfraw der konigin von himelreich. ein maget ist ein swester der engel. ein maget ist ein schon aller heiligen ein maget ist ein frewde alles himelischen hers ein maget ist ein liecht tragerin in dem himel. ein maget ist ein gezierde des menschen. ein maget ist frawe aller creature $\left(98^{\mathrm{n}-\mathrm{t}}\right)$.

Allein schon dieser analoge Lobpreis der Jungfräulichkeit gibt Anlaß zu der Vermutung, daß die Beziehung Christus - Johannes tatsächlich Modell ist für die Selbstinterpretation der jungfräulichen Religiosen in den frühen Dominikanerinnenklöstern und geistlichen Gemeinschaften im Bodenseeraum.

An Bernhards Predigt schließt sich eine Vita des Evangelisten an $\left(48^{\text {rb }}-61^{\text {vb }}\right)$, die mit Vinzenz von Beauvais einer Autorität des eigenen Ordens zugeschrieben wird. Diese Zuweisung ist bemerkenswert, denn seit den späten fünfziger Jahren lag die Legenda Aurea, die Legendenkompilation des Dominikaners Jacobus a Voragine, vor, die hier 
als Quelle ausgeschieden werden kann. ${ }^{42}$ Die Verbreitung der Legenda Aurea ist - quantitativ und geographisch breit gestreut - seit den achtziger Jahren des Jahrhunderts gut dokumentierbar. ${ }^{43}$ Ein Basler Druck aus dem Jahre 1481 enthält 'Opuscula' des Vinzenz von Beauvais, u.a. auch ein Liber de sancto Johanne evangelista. Die Autorschaft Vinzenz' kann allerdings nicht als zweifelsfrei gesichert gelten. Edition und Untersuchung fehlen. ${ }^{44}$

Erst nach diesem durch Autoritäten gesicherten Vorlauf beginnen die Texte der Konstanz-Züricher Dominikaner mit der Jakobus-Predigt Konrads von Liebenberg, in deren Mittelpunkt nicht Jakobus selbst, sondern der Evangelist steht $\left(61^{\text {vb }}-69^{\text {rb }}\right)$. Sie bietet hauptsächlich familiengeschichtliche Ergänzungen zur vorausgehenden Johannes-Vita. Daneben enthălt sie marginale Hinweise auf das Verhältnis von Philosophie und Theologie bei den Dominikanern. 'darvmb wais plato minner den ein esel' $\left(65^{\text {b }}\right)$ schreibt Konrad und meint damit, 'das er von seiner naturlicher weishait vnd mit seinen haidenischen listen erkant das ein angenge mus sein von dem alle ding geschaffen seint $\left(65^{\circ}\right) \ldots$ Aber das angenge ist der vater vnd $\mathrm{dz}$ wort der sun vnd der heilig gaist ir baider minne vnd das drey person seint in der driualtikait des gedacht plato nie.' Mit anderen Worten: Die Naturdinge sind den heidnischen Philosophen erkennbar gewesen, die celestia nicht. ${ }^{45}$

Auf eine anonyme Johannespredigt $\left(69^{\text {rb }}-79^{\text {ra }}\right)$ folgen dann die Texte Heinrichs von Schaffhausen $\left(79^{r}-89^{r a}\right)$ und Rudolfs von Klingenberg $\left(89^{\mathrm{m}}-97^{\mathrm{tb}}\right)$. Heinrichs Predigt bietet dabei einen zusätzlichen Hinweis auf die Verwendung dieser Texte in der cura, denn bei ihm werden Maria zur Priorin und Johannes zum Prior von 72 Jungfrauen in Jerusalem:

12 Barbara Fleith, Studien zur Überlieferungsgeschichte der lateinischen Legenda Aurea, Bruxelles 1991 (Subsidia Hagiographica 72), S.16.

${ }^{43}$ Ebda, S.367-403.

"Rudolf Weigand, Vinzenz von Beauvais. Scholastische Universalchronistik als Quelle volkssprachiger Geschichtsschreibung, Hildesheim/Zürich/New York 1991 (Germanistische Studien und Texte 36), S.18.

${ }^{45}$ Langer, Mystische Erfahrung (Anm. 20), S.1-3. 
so fur er dan vber mer ze ierusalem da het er vnser frawen in ainem hausse vnd gab ir sant jgnacius zu ainem kapplan vnd ze ainem sunderlichen screiber der was sein iunger vnd was ain heilig bischoff vnd hat bey ir zwuvndsibenczig megde der besten von dem lande vnd gab den gewant vnd speise vnd alles des sie bedorften vnd was vnse frawe da priorin vnd sannt johannes prior $\left(86^{\text {t) }}\right)$.

Die folgende Agnes-Predigt ist wiederum anonym (97 $\left.{ }^{\text {th }}-103\right)$. Ihre vermittelnde Funktion zwischen den Predigten auf den Evangelisten und den Baptisten nimmt sie wahr, indem sie die beiden Johannsen durch ein Symbol verbindet, das beiden gemeinsam ist, durch das Lamm. Gleichzeitig treten Evangelist und Täufer als Autoritäten in dieser Predigt auf, die die Sonderstellung des Jungfrauenstandes autoritativ absichern. Die erste Predigt auf den Täufer wird dann Hugo von Konstanz zugeschrieben $\left(103^{\text {rt }}-112^{\text {vb }}\right)$, die beiden folgenden bleiben wiederum ohne Verfassernennung $\left(112^{\mathrm{vb}}-120^{\mathrm{vb}}, 120^{\mathrm{vb}}-130^{\mathrm{ra}}\right)$.

Lateinische Vorlagen für die Predigten konnten bisher noch nicht nachgewiesen werden, sind aber nicht auszuschließen, da viele initiengleiche Texte unediert und daher schwer zugänglich sind. Da Texte ohne Verfasserangabe immer auf Texte mit Verfasserangabe folgen, ist die Zuweisung der anonymen Texte an die vorausgehenden Autoren zu erwägen.

Ich fasse zusammen: Abgesehen von den zuletzt genannten Fragen dokumentiert die Abfolge der Texte eine geplante Anlage: Auf biblische und pseudobiblische Texte folgen zwei Autoritäten, Bernhard und Vinzenz, dann die Texte der Konstanz-Züricher Dominikaner, die die Sequenzen zum Evangelisten und zum Täufer geschickt mit einer AgnesPredigt verknüpfen. Trotz einiger Unsicherheiten spricht dieser Befund für eine einheitliche Entstehung des gesamten Textcorpus um $1300{ }^{46}$

\footnotetext{
${ }^{46}$ Gewisse Unsicherheiten hinsichtlich der Datierung aller Texte in das 4. Viertel des 13. Jahrhunderts bleiben bestehen. Dies gilt insbesondere für die Übersetzung der Apokalypse. Andererseits gibt es keine Hinweise auf die Verwendung der Legenda Aurea; darin wäre mit aller gebotenen Vorsicht ein Indiz für frühe Entstehung der Texte zu sehen. Der 2. Teil $\left(132^{r}-245^{r}\right)$ der Handschrift enthält die Hieronymusbriefe in der Übersetzung des Johannes von Neumarkt. Er ist durch Lagengrenze vom Johannescorpus getrennt und kein integraler Bestandteil, sondern
} 
In der vorliegenden Form kann die Zusammenstellung als Johanneslibellus bezeichnet werden. ${ }^{47}$

\section{Die Predigt Rudolfs von Klingenberg}

Rudolfs Predigt erstreckt sich über neun Folioblätter und ist damit repräsentativ in der Quantität für die vorliegende Textsammlung ( $89^{\text {ra }}$ $97^{\text {tb)}}$ ). Sie soll hier exemplarisch für die Predigten der gesamten Sammlung vorgestellt werden.

Die Predigt folgt dem Bibelwort 'Conversus Petrus vidit illum discipulum quem diligebat Iesus sequentem et recubuit in cena super pectus eius' (Jo 21, 20). ${ }^{48}$ Nach einem Prolog, in dem die Bedeutung des Evangelisten mit der sprachlichen Ohnmacht des Predigers konfrontiert wird, beginnt die Predigt mit einer Paraphrase des Initiums, an die eine dreigliedrige Disposition mit den folgenden Punkten anknüpft: 'die sunderlich minne' (die besondere Liebe) Christi zu Johannes, 'die wirdikait seines lones' (die Angemessenheit seines Lohns) und 'die minnikliche lere' (die Liebeslehre) des Johannes. Die einzelnen membra werden nicht ausgewogen behandelt. Von $89^{\text {tb }}$ bis $96^{\text {th }}$, also über sieben Blätter hinweg, steht die besondere Vorliebe des Herrn für Johannes im Mittelpunkt. Subpartitiones gliedern diesen Teil und behandeln Johannes an der Brust des Herrn, Johannes unter dem Kreuz, Johannes bei Maria an Sohnesstatt und die vorzeitige Verklärung des Johannes. Den Schluß der Predigt bilden kurze Bemerkungen zu Werk und

nur additiv hinzugefügt. Eine Durchsicht der gesamten Überlieferung der deutschen Hieronymusbriefe erweist die Kombination mit einem Johannes-libellus als einmalig und bestätigt damit die additive Überlieferungsform.

47 Weitere Johannes-libelli bieten Handschriften aus Bamberg, Basel und Karlsruhe. Sie stammen alle aus dem 15. Jahrhundert und weisen im Bereich der Predigten keine Parallelüberlieferung zu unserem Codex auf. In dem Bamberger und Basler Codices werden die Johannestexte mit Viten der Schwestern von Töss und Katharinental bei Diessenhofen kombiniert: Bamberg, SB, Hist. 152 (Johannes Baptista); ebda, Hist. 153 (Johannes Evangelista); Basel, UB, A.VI.38; Karlsruhe, Badische LB, St. Peter pap. 21.

48 Zur Liebesthematik im Johannes-Evangelium vgl. jetzt Sjef van Tilborg, Imaginative Love in John, Leiden/New York/Köln 1993 (Biblical Interpretation Series 2), hier S.77-110, 154-157. 
Himmelfahrt des Johannes. Die Konzentration auf die hier genannten Schwerpunkte ist für alle Predigten der Sammlung charakteristisch. Ergänzend treten hinzu: die Auslegung des Adlersymbols und die Martern. Die Missionstätigkeit wird nur in der Legende ausführlicher gewürdigt.

Diese thematische Konzentration auf die besondere Beziehung Christi zum Evangelisten wird von Rudolf durch profane Minnesentenzen hervorgehoben:

wann das geschihet auch an lieplicher minne so ein mensche mit dem andern lieplich vnd minniklich redet das im sein hercz zerflusset von minne gegen seinen lieben. $\left(92^{\text {tb }}\right)$.

Dieser minnedidaktische Einschub dient in Anknüpfung an den literarischen Liebesdiskurs der Erläuterung des Bibelworts Jo 13, 23: 'Erat ergo recumbens unus ex discipulis eius in sinu Iesu quem diligebat Iesus'. In der Auslegungstradition dieser Bibelstelle ist das Johannes Evangelista, 'der geminnte des herrn', der Sohn des Zebedeus und der Maria Salome, der Bruder Jakobus' des Ålteren. Die folgende Auslegung dieser Minnesentenz gibt dem Text dann wieder die erwartete spirituelle Ausrichtung:

Dis geschihet an lieplicher minne so mercke ein iglich mensche wie vil billicher es denn geschehe an der gotlichen minne wann do der ausflus des lebendigen brunnen samenthaft flos in sant Johannes da was billich das sein sele vnd sein hercz zerflos von minne davon macht er wol sprechen als da gescriben stet in der minnebuch Mein geminter hat zu mir geredet. davon ist mein sele vnd mein hercze hin fliessenden worden von sussikait vnd von minne $\left(92^{\text {th-nan }}\right)$.

Diese Besonderheit seiner Predigtweise zeigt sich auch im Rückgriff auf allgemeine Lebenserfahrungen:

vnser herre der tet recht als ein vater der lieber kinde vil het vnd aus den allen ains minnet das im sunderlich lieb ist vnd dem er vsgenomene zaichen der minne erzaiget fur die andern alle $\left(90^{\text {va }}\right)$ - Ir wissent wol so ein mensch susses tranck getrincket das im lustlich ist. so begert es sein alle zeit ie mer vnd ie mer $\left(93^{\mathrm{n}}\right)$. 
Gleichzeitige deutschsprachige Johannes-Predigten dominikanischer Provenienz, die zum Vergleich herangezogen werden könnten, sind nicht überliefert. Zeitlich vorausgehende Predigten auf den Evangelisten und den Täufer finden sich in den frühen deutschen Predigthandbüchern, die dem Seelsorger im Sinne eines 'Dormi secure' die Arbeit erleichtern sollten.

Aus Raumgründen beschränke ich mich auf die Angabe der $T$ - und Veröffentlichungsnummer nach Karin Morvay und Dagmar Grube: Bibliographie der deutschen Predigt des Mittelalters. Veröffentlichte Predigten, München 1974 (MTU 47): Speculum Ecclesie T 9, Nr. 49, S. 27-29 (Joh. Ev.); S. 29-30 (Joh. Ev.); S. 80-81 (Joh. Bap.). Grieshabersche Sammlung I T 10, Nr. 54, S. $445-447$ (2 x Joh. Bap.). Mitteldeutsche Predigten T 11-14, Nr. 55, S. 23f., vollst: Berlin, SBBPK, Ms. germ. qu. 2025, 279v-282v (Joh. Bap.); ebda, 252r-255v (Joh. Ev.). Rothsche Sammlung T 16, Nr. 61, S. 21f. (Joh. Ev.). Leipziger Sammlung T 17, Nr. 64, S. 60-63 (Joh. Bap.); T 15, Nr. 60, S. 77-81 (Joh. Ev.); T 17, Nr. 64, S. $185-187$ (Joh. Ev.); T 17, Nr. 64, S. 203f. (Joh. Bap.); T 17, Nr. 64, S. 258-261 = T 19, Nr. 72, S. 233-236 (Joh. Ev.); T 17, Nr. 64, S. 333-336 (Joh. Bap.). Schlägler Bruchstücke T 21, Nr. 74, S. 18-23 (Joh. Bap.). Oberaltaicher Sammlung T 23, Nr. 76, S. 21f. (Joh. Ev.). Priester Konrad T 25, Nr. 80, S. $17-20=$ T 27, Nr. 85, S. $404-405$ (Joh. Ev.); T 25, Nr. 80, S. $190 f$. (Joh. Bap.); T 25, Nr. 80, S. 192f. (Joh. Bap.); T 25, Nr. 80, S. 209.211 (Decollatio). Kuppitsch'sche Predigtsammlung T 34, Nr. 100, Sp. 411-413 (Joh. Ev.); T 34, Krakau, Biblioteka Jagielloriska, Ms. germ. qu. 484, 52r/v, 61v-63r (Joh. Bap.). Bruchstïcke des 12. Jahrhunderts aus Wilhelm Wackernagels Altdeutschen Predigten T 36, Nr. 91, S. 33f. (Joh. Ev.). St. Pauler Predigten T 39: The St. Pauler Predigten, ed. by Norman E. Whisnant, Chapel Hill, Ph.D. 1978, S. 59-64 (Joh. Ev.); ebda, S. 259-266 (Joh. Bap.). Mettener Sammlung T 40, Nr. 112, S. 213-215 (Decollatio). Schwarzwälder Predigten T 60, Sanctorale: Pommersfelden, Gräflich Schönbornsche Bibliothek, Ms. 101, 24rb-29vb (Joh. Ev.); ebda, 98ra-104ra (Joh. Bap.). Schmids bairische Predigtsammlung s. hierzu Hans Ulrich Schmid, 'Eine bairische Predigtsammlung des späten 13 Jahrhunderts', in Die deutsche Predigt im Mittelalter, hrsg. von Volker Mertens und Hans-Jochen Schiewer, Tübingen 1992, S. 55-81, hier S. 67. Die Buchwaldschen Heiligenpredigten werden nicht berücksichtigt, da es keine Anhaltspunkte mehr für deren Entstehen im 13. Jahrhundert gibt.

Charakteristisch ist hier vielfach die Kürze und die Reduzierung des Gehalts auf die legendarischen Gesichtspunkte. Ich zitiere aus der Leipziger Sammlung eine Predigt auf den Evangelisten:

Johannes apostolus et ewangelista etc. Mine vil lieben, hute ist der tach des gaten sente Johannis ewangelisten der unsers herrin trat was, dem unser herre des gonde daz er rawete uf sinen brústen des nachtes ... der selbe gotis trat der was ein ewich magt, dem bevalch unser herre sine libe muter ... der dinte er di wile daz si in ertriche was. ... do vur er zu Kriechen und predigete gotis wort und bekarte da als 
vil volkes, ...99

Die Minnethematik tritt in diesen Berichten weitgehend in den Hintergrund bzw. beschränkt sich auf die Stichworte 'Lieblingsjünger' und 'ewige maget'; die St. Pauler Predigten nähern sich mit der einmal belegten Formel 'erweltiv meit' (Whisnant, S. 60) am weitesten der sponsa-Thematik. Die erzăhlerische Füllung der Predigt erfolgt durch Berichte über apostolisches Wirken, Wunder und Martern, wie sie im Bereich der Heiligenpredigten typisch sind. ${ }^{50}$ Erwähnt werden häufig die Bekehrung der drei Jünglinge und eines Pflegekinds, die Erweckung von Toten (Thadeus, Drusiana etc.), die Umwandlung von Holz und Steinen in Gold, das Ölbad, der Schierlingsbecher und das Exil auf Pathmos sowie - selbstverständlich - die leib-seelische Himmelfahrt und das im leeren Grab aufwallende Himmelbrot.

Diese Schlaglichter sollen genügen, um die inhaltliche Sonderstellung der Johannespredigten in der vorliegenden Textsammlung deutlich zu machen. Eine Einbindung in vorgängige Traditionen der deutschsprachigen Predigt ist nicht möglich.

\section{Die Stellung der Textsammlung innerhalb des dominikanischen Schriftums}

Mit Eckhart und den Nonnenviten fassen wir den bisher gültigen Ausgangspunkt dominikanischen Schrifttums im oberdeutschen Raum. Noch Cramer schreibt in seiner Literaturgeschichte (1990): 'Bestimmende Gestalt der spătmittelalterlichen deutschen Mystik ist Meister Eckhart. Durch ihn wird der Dominikanerorden zur Heimat mystischen Denkens, nachdem das 13. Jahrhundert von der franziskanischen Mystik

\footnotetext{
${ }^{49}$ Anton E. Schönbach, Altdeutsche Predigten I, Graz 1886, S.185f.

so Eine qualitative Ausnahme sind die Schwarzwälder Predigten, die etwa gleichzeitig mit den hier vorgestellten Predigten entstanden. Ihre Vorlage war die Legenda Aurea des Jacobus a Voragine. Vgl. Werner Williams-Krapp, 'Das Gesamtwerk des sog. "Schwarzwälder Predigers"', ZfdA 107 (1978), 50-80.
} 
geprägt war. ${ }^{\$ 1}$ Das stimmt, soweit es die Bedeutung Eckharts betrifft, muß aber relativiert werden im Hinblick auf ein franziskanisches 13. und ein dominikanisches 14 . Jahrhundert. Denn unsere Textsammlung gibt Einblick in dominikanisches Schrifttum des späten 13. Jahrhunderts in einer zentralen Literaturlandschaft des deutschen Sprachraums. Bisher waren die dokumentierten Spuren der volkssprachlichen dominikanischen cura im 13. Jahrhundert sehr spärlich. Konserviert vor allem in den Predigtsammlungen des beginnenden 14. Jahrhunderts, d.h. in den Kölner Klosterpredigten ${ }^{52}$ und im Paradisus anime intelligentis. ${ }^{53}$ Dort dominieren Kölner Dominikaner und Dominikaner aus der Saxonia, meist eine Generation jünger als unsere Konstanzer und Züricher Prediger. Eine der wenigen Ausnahmen macht der mit einer Predigt in den Kölner Klosterpredigten vertretene Ulrich Engelberti aus Straßburg, der von 1271 bis 1277 Provinzial der Teutonia war und somit von Profil und Alter her neben Hugo von Konstanz tritt. ${ }^{54}$ Weitere Spuren bietet das X-Corpus der Berthold-Überlieferung, in dessen Kontext ein 'Bruder Albrecht sant Dominicus orden' mit drei Predigten und ein 'Bruoder Albreht der lesemeister' mit einer Predigt überliefert werden. ${ }^{53}$ Die für den oberdeutschen Raum im 13. Jahrhundert geschlossenste Textgruppe stammt nun von den vorgestellten KonstanzZüricher Dominikanern. Sie werden dadurch zu den unmittelbaren

${ }^{51}$ Thomas Cramer, Geschichte der deutschen Literatur im späten Mittelalter, München 1990, S.178. Ähnliche Äußerungen finden sich bei Kurt Ruh, 'Geistliche Prosa', in Europäisches Spätmittelalter, hrsg. von Willi Erzgräber, Wiesbaden 1978 (Neues Handbuch der Literaturwissenschaft 8), S.565-605, hier S.582, 593, 594; Alois M. Haas, 'Deutsche Mystik', in Die deutsche Literatur im späten Mittelalter 1250-1370. T.2: Reimpaargedichte, Drama, Prosa, hrsg. von Ingeborg Glier, München 1987 (Geschichte der deutschen Literatur von den Anfängen bis zur Gegenwart 3/2), S.234-303; Georg Steer, 'Geistliche Prosa', in ebda, S.305-370. Vgl. auch Muschg, Mystik (Anm. 17), passim.

${ }^{52}$ Volker Honemann und Dagmar Ladisch-Grube, in ${ }^{2} V L$ V (1985), 49-54.

${ }^{53}$ Kurt Ruh, 'Deutsche Predigtbücher des Mittelalters', in K.R., Schriften (Anm. 11), S.296-317, hier S.312-317.

s4 J. Daguillon, 'Ulrich de Strasbourg, prédicateur. Un sermon inédit du XIII' siècle', in La Vie Spirituelle, 1926, Supplément, 8498.

ss Dieter Richter, Die deutsche Überlieferung der Predigten Bertholds von Regensburg. Untersuchungen zur geistlichen Literatur des Spätmittelalters, München 1969 (MTU 21), S.29, 32f. Die Texte der genannten Dominikaner gehören zur Gruppe $\mathrm{X}^{\mathrm{n}}$, deren Entstehung im 13. Jh. nicht gesichert ist (ebda, S.70-72 u. 78). 
Vorläufern Heinrich Seuses, der um 1310 in das Konstanzer Predigerkloster eintrat und nach abgeschlossenem Studium 1327 in das Bodenseekloster zurückkehrte. Seuse gilt zwar als Schüler Eckharts, Einfluß der hier vorgestellten Texte auf seine Schriften muB nun aber erwogen werden.

Ungefilterte, d.h. nicht durch Beichtvater oder Lektor redigierte Reflexe der Vorliebe für den Evangelisten bietet Elsbeth von Oye in ihren eigenhändig in Ötenbach niedergeschriebenen Offenbarungen aus dem 2. Viertel des 14. Jahrhunderts. ${ }^{56}$ Ein eigenständiger Faszikel mit Konzeptcharakter ist ausschließlich dem Lieblingsjünger Christi gewidmet und enthält Offenbarungen des Herrn über den Evangelisten und teils Dialoge des Evangelisten mit Elsbeth selbst. Die Funktion des Johannes als Vermittler der unio wird darin deutlich ausgesprochen:

Dizú wort wurden zi mir gisprochin

von sant Johannes ewangelista:

'Alz ich in miner mater zeinem

phleger gab uf ertriche, also

habe ich in dir gegebin, daz

er si ein miteler zwischen

mir unt diner sele unt

er alle zit gälich unt mit

spilinder vróde mir inklin-

gil in min veterlich herze

alle din tabunge in der

brinnenden brunst siner min-

nebiwegunge unt dem min-

eklichin zarte, alz ich in

mir selber habe irwelt zi spi-

so Bezüge in der publizierten Viten- und Offenbarungsliteratur bleiben hier unberücksichtigt. Speziell zu Katharinental vgl. Arno Borst, Mönche am Bodensee, Sigmaringen 1978, S.284-301. 
lender vrode in miner ewikeit. ${ }^{57}$

(Zürich, ZB, Rh 159 , S. 145)

Dieser Funktion wird der Evangelist auch gerecht, wenn er sich mit den folgenden Worten Elsbeth offenbart:

'Darumbe habe ich dir gi-

vordert von dem veterlichin herzen

die blatigisten glicheit sines

kruzgeten suns, daz du in

dem minnewallinden blâte

sins suns alle zit ein wi-

derfliezzin habist in den tief-

stin grunt siner gotlicher nature.'

(Zürich, ZB, Rh 159, S. 152)

Darüber hinaus stellt sich im Hinblick auf unsere Autorengruppe erneut die Verfasserfrage für das Solothurner oder Marchwart-Biberli-Legendar. Die Schlußbemerkung zu den ersten 26 Heiligenlegenden charakterisiert Biberli nur als Begutachter, nicht als Übersetzer der Sammlung:

Dis heiligen sint bewerte heiligen ... vnd wart ir vil ze tútsch braht ab einem vil alten buche. vnd b́berlas es da bi vil alles ein wiser Lesmeister bredier ordens bruder Marchwart Biberli. dem gar kunt ist vmb der heiligen legende. der sprach daz es ganz vnd gerecht also wer..$^{58}$

Ein Blick auf die leider immer noch nicht edierte Sammlung zeigt ähnliche inhaltliche Strukturen, wie wir sie schon in der JohannesPredigt Rudolfs von Klingenberg kennengelernt haben, und zwar geradezu programmatisch im Prolog. Ebenso wie oben die Beziehung

${ }^{57}$ Ich danke Wolfram Schneider-Lastin (Basel) für die Überlassung seiner noch ungedruckten Edition der Offenbarungen, nach der ich hier zitiere. Vgl. dazu ders., 'Das Handexemplar einer mittelalterlichen Autorin. Zur Edition der 'Offernbarungen' Elsbeths von Oye', editio 8 (1994). Zum bisherigen Forschungsstand s. Peter Ochsenbein, 'Die Offenbarungen Elsbeths von Oye als Dokument leidensfixierter Mystik', in Abendländische Mystik (Anm. 14), S.423-442.

${ }^{58}$ Zitiert nach Geith, 'Biberli' (Anm. 14), S.10. Wallach-Faller, Psalter (Anm. 14), S.90-109 versucht, in einer komplizierten Argumentation auf die Verfasserschaft Biberlis zu schließen. 
Christus-Johannes im Rückgriff auf profane Minneerfahrung beschrieben worden ist, geschieht dies auch hier im Zusammenhang mit der Auslegung von $\mathrm{Cn} 2,5$ :

Swer an sin lieb nit sur mag. Vnd im doch sin lieb nit ze liebe werden mag. der mus dike ein minne siech herze tragen.

Die Wendung ins Geistliche schließt unmittelbar an:

Dis geschicht ${ }^{\circ} \mathrm{ch}$ gar dike einer Got minnender siechen sele. Dv ane das stisse gotlich lieb nit mag stan. vnd es doch in disem zite nach herzen girde nit mag haben. $D$ j siget also herzlos da hin. ${ }^{59}$

Bemerkenswert ist auch, daß das Solothumer Legendar auf einem 'légendaire traditionelle' beruht und ebenso wie unser Textcorpus keine Berührungspunkte mit der Legenda Aurea aufweist. Selbstverständlich reichen diese Hinweise nicht aus, um Rudolf von Klingenberg als Übersetzer oder Mitautor des Solothumer Legendars zu erweisen, aber die Verwandtschaft unserer Predigten zum Legendar wird deutlich und erweitert die Verfasserdiskussion um den Kreis der hier vorgestellten Autoren.

Der Umfang der literarischen Tätigkeit der Konstanz-Züricher Dominikaner ist bisher nur in Umrissen erkennbar, seine spezifische Ausrichtung aber deutlich. Das Thema göttlicher Minne wird am Beispiel der beiden Johannsen abgehandelt, die brautmystische Komponente tritt dabei in den Hintergrund, wird aber im Sponsa-Motiv des an der Brust des Herrn ruhenden Evangelisten wieder aufgenommen.

Diese Motivik korrespondiert mit einer im südwestdeutschen Raum um 1300 gepflegten Johannes-Verehrung, wie sie in den ChristusJohannes-Bildgruppen des alemannischen Raums auch in der darstellenden Kunst zum Ausdruck kommt. ${ }^{60}$ Von den 25 erhaltenen

${ }^{59}$ Zitiert nach Wallach-Faller, 'Dominikanerinnen-Legendar' (Anm. 14), S.389.

6o Rainer Hausherr, 'Über die Christus-Johannes-Gruppen. Zum Problem "Andachtsbilder" und deutsche Mystik', in Beiträge zur Kunst des Mittelalters. Fesichrift für Hans Wentzel zum 60. Geburtstag, Berlin 1975, S.79-103. Zuletzt E.M. Vetter, 'Das 
plastischen Gruppen stammen 14 aus dem 14. Jahrhundert und - sofern die ursprüngliche Bestimmung bekannt ist - ausschließlich aus Frauenklöstern, vorrangig aus Häusern der Dominikanerinnen. ${ }^{61}$ Die Visualisierung der Beziehung Christus-Johannes - hier interpretiert als Modell der Selbstinterpretation der jungfräulichen Religiosen korrespondiert mit den vorgestellten Texten, hatte entscheidenden Einfluß auf die Frömmigkeitspraxis und regte visionäre Gottesbegegnungen der Schwestern an. ${ }^{62}$ Berücksichtigt man nun diese Jesus-Johannes-Gruppen, rückt ein von den Konstanzer Dominikanern betreutes Kloster in den Mittelpunkt des Interesses, nämlich Katharinental bei Diessenhofen: Dieses Kloster besaß eine berühmte JesusJohannes-Gruppe des Meisters Heinrich aus Konstanz, die kurz nach 1300 entstand (Abb. 2). Zugleich verehrten die Schwestern den Täufer, dem einer ihrer Altäre geweiht war. ${ }^{63}$ Dieser Doppelkult führte sogar zu einer Aufspaltung des Konvents in 'Evangelistinnen' und 'Baptistinnen'. ${ }^{\text {'4 }}$

Christus-Johannesbild der Mystik', in Mystik am Oberrhein, Ausstellungskatalog, Freiburg i.Br. 1978, S.37-50.

${ }^{61}$ Hausherr, 'Christus' (Anm. 60), S.81.

${ }^{62}$ Über den Zusammenhang der Auditionen und Visionen der Religiosen und Schwestern mit der künstlerischen Ausstattung der Konvente und Handschriften vgl. die wegweisenden Arbeiten von Jeffrey F. Hamburger, 'The Visual and the Visionary. The Image in Late Medieval Monastic Devotions', Viator 20 (1989), 161206, hier 173f.; ders., "The Use of Images in the Pastoral Care of Nuns. The Case of Heinrich Suso and the Dominicans', The Art Bulletin 71 (1989), 20-44, und zuletzt ders., 'Art, Enclosure and the Cura Monialium. Prolegomena in the Guise of a Postscript', Gesta 31 (1992), 108-134.

${ }^{63}$ Hausherr, 'Christus' (Anm. 60), S.82-84; Albert Knoepfli, Die Kunstdenkmäler des Kantons Thurgau, Bd IV: Das Kloster St. Katharinenthal, Basel 1989, (Die Kunstdenkmäler der Schweiz 83), S.231ff. Zugleich besaß das Kloster eine lebensgroße Plastik des Johannes Baptista, die um 1300 möglicherweise ebenfalls in der Heinzelin-Werkstatt entstand (ebda, S.226f.).

${ }^{64}$ Ebda, S.227; Albert Knoepfli, 'Geschichte des Klosters St. Katharinental unter besonderer Berücksichtigung der Gründung und Anfangszeit', in Das Graduale von Sankt Katharinental. Kommentar, Luzern 1979, S.1-66, hier S.60. 
Abb. 2 Meister Heinrich von Konstanz, Jesus-Johannes-Gruppe, nach 1300; Nußbaumholzblock, $141 \times 73$ x $48 \mathrm{~cm}$. Antwerpen, Museum Mayer van der Bergh.

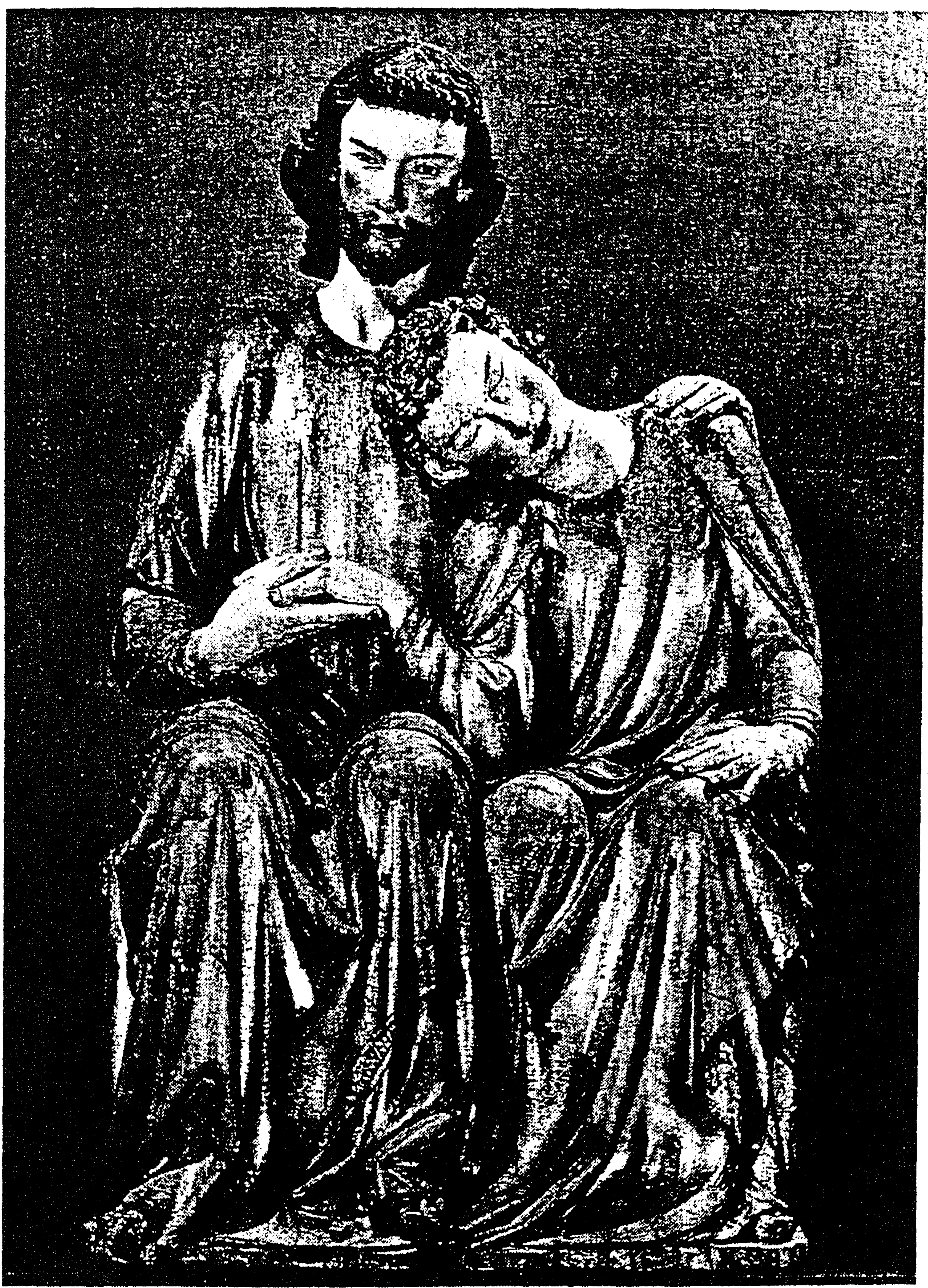


Der Platz in der literarischen Landschaft am Bodensee

Dieser Disput um den Vorrang von Evangelist und Täufer blieb nicht auf die klösterliche Klausur beschränkt, denn aus dem frühen 14. Jahrhundert wird ein Streitgedicht Heinzelins von Konstanz überliefert, das dieses Thema 'Von den zwein Sanct Johansen' aufgreift:

\section{0}

In einem richen kloster waz

so vil der klosternunnen, die sich gote verre baz dann zu der werlt versunnen. die eine sank, die ander laz sam klosterliute kunnen.

11

In herzen kondenz alle sampt gar inneclichen horden, wie sie begingen gotes ampt und hielten wol iren orden; wann wer sich sinez ordens schampt, des gutet ist erworden.

12

Von in wart nieman denne got gemeinet noch geminnet, sie leistent gerne sin gebot und warent dez versinnet, daz der gelaub ist halber tot, wer niht der werk beginnet.

13

Ir klosterzuht in wirde schein fur ander klosterleute, wann daz sich hup enzwischen zwein ein krieg, der wert noch heute. die hullent beide niht inein als ich euch hie beteute. 
14

Die einen hort man brüfen sant

Johansen baptisten,

der gotes toufer ist genant,

die andern ewangelisten. ${ }^{65}$

Heinzelin schrieb sein Streitgedicht vermutlich für Albrecht V. von Hohenberg, der am Beginn des 14. Jahrhunderts Domherr in Konstanz war. Die Überlieferung ist auf die 1. Hälfte des 14. Jahrhunderts beschränkt und vollzieht sich ausschließlich in deutsch-lateinischen Mischhandschriften aus dem klerikalen Bereich, deren berühmteste wohl Hausbuch und Manuale des Würzburger Protonotars Michael de Leone sind. ${ }^{66}$ Diese Spur führt uns zurück an den bischöflichen Hof und damit in die 'literarischen Salons' von Zürich und Konstanz. Ein zweites Streitgedicht Heinzelins für denselben Auftraggeber behandelt in deutscher Sprache erstmals die alte Streitfrage, ob der miles oder clericus der bessere Liebhaber sei. Bemerkenswert ist hieran nicht nur das offene Ende, sondern auch die argumentative Ausdifferenzierung des Klerus im Hinblick auf die Eignung als Liebhaber. Die Vertreterin der clerici schlägt am Ende des Disputs einen Haken und macht die Eignung vom Weihegrad abhăngig:

jo mein ich solicher pfaffen niht, di man di messe sprechen siht, ich mein, di pfaffen sint genant

6s Heinzelin von Konstanz, in Die kleineren Liederdichter des 14. und 15. Jahrhunderts. Bd. 1. Adam von Fulda - Heinzelin von Konstanz, hrsg. von Thomas Cramer, München 1977, S.375-392, hier S.377f. Als literarischer Stoff taucht der Streit zwischen 'Evangelistinnen' und 'Baptistinnen' erstmals bei Caesarius von Heisterbach (Dialogus miraculorum, hrsg. von J. Strange, Köln/Bonn/Brüssel 1851, dist. VIII, c. LI) auf, dann wiederum bei Franko von Meschede. Ruh ( ${ }^{2} V L$ II [1980], 831f.) verweist auf eine deutschsprachige Fassung in dem schon oben genannten Johannes-Libellus aus Bamberg, SB, Hist. 153, 137 $7^{\mathrm{T}}$-139.

${ }^{66} \mathrm{Vgl}$. Gisela Kornrumpf, in ${ }^{2}$ VL VI (1987), 491-503. Hausbuch des Michael de Leone, München, UB, cod. $2^{\circ} \mathrm{ms} 731,270^{\circ}-273^{\circ}$, ca. 1344-1354; Manuale des Michael de Leone, Würzburg, UB, cod. M.p.misc.f.6, 64'-66', ca. 1342/43-1354; Bern, Burgerbibliothek, cod. Nr. 260, 152r-155r, Mitte 14. Jh. Die Berner Hs. überliefert auch Lieder Johannes Hadlaubs und wird in Zusammenhang gebracht mit dem Historiographen und Protonotar des Basler und Straßburger Bischofs Matthias von Neuenburg. Vgl. dazu Peters, Stadt (Anm. 4), S.141-154, bes. S.154 Anm. 54. 
und doch nit hoher wihe hant.

si sint der pfafheit zu gezelt

um niht wen um ir pfeflich gelt.

di priesterlichen pfaffen

sol man ir ding lan schaffin.

derselben ich niht mein;

ich mein, di enklein

sint pfaffen als du merkest mich wol. ${ }^{67}$

Ohne daß ein direkter Bezug zum Konstanzer Bischof Heinrich von Klingenberg herstellbar wäre - dies scheitert schon aus chronologischen Gründen -, korrespondiert diese Volte des Streitgedichts mit der Biographie Heinrichs, der 1289 von der Pflicht zum Empfang höherer Weihen dispensiert wurde. Seine Tochter war dann Schwester im Dominikanerinnenkloster Habsthal und wurde testamentarisch bedacht. ${ }^{68}$

Gleichzeitig schreibt Johannes von Konstanz eine der ersten Minnelehren, die in der vielleicht aus Konstanz stammenden Weingartner Liederhandschrift B erstmals, wenn auch anonym, überliefert wird. Im Konstanzer Haus zur Kunkel entstanden um 1320 Fresken zu Wolframs Parzival. Zu diesem Zeitpunkt befand sich das Haus im Besitz der Chorherren an der Kirche St. Johann, die den 'Geschwisterheiligen', d.h. beiden Johannsen geweiht war. Das Haus wurde von Stiftsgeistlichen bewohnt. ${ }^{69}$ Über den Protonotar Heinrich Offenbach von Isny des Konstanzer Bischofs Nikolaus I. von Frauenfeld (1334-1344), Kanonikus an St. Stephan und späteren Domherren berichtet die Zimmerische Chronik: 'Auch het derzeit bischof Niclas von Costanz ain secretari gehapt, herr Hainrich, der ist gleichfals mit den deutschen lieder und gerüempten gedichten umbgangen, zu vermuten, sie haben dozumal nit grösser oder mer gescheft gehapt, sonder nur de faire bon

${ }^{67}$ Heinzelin von Konstanz (Anm. 65), S.393-403, hier S.401; Ingrid Kasten, Studien zu Thematik und Form des mittelhochdeutschen Streitgedichts, Phil. Diss. Hamburg 1973, S.86-90.

Die Manessische Liederhandschrift (Anm. 1), S.24.

${ }^{69}$ Bernd Schirok, 'Die Parzivaldarstellungen in (ehemals) Lübeck, Braunschweig und Konstanz', Wolfram-Studien 12 (1992), 172-190, hier 184-186. 
temps' ${ }^{70}$ Er darf als Urheber und Schreiber der verschollenen Liederhandschrift $\mathrm{X}$ gelten. Diese thematische Überschneidung verschiedener Literaturkreise kann nicht überraschen, wenn man die Sozialstruktur der Dominikanerkonvente in den Blick nimmt, denn nicht nur die Lektoren und Beichtväter entstammten dem stadt- und landsässigen Adel der Region, sondern auch die Gründer der Frauenklöster und die Konventualinnen selbst: $\mathrm{Zu}$ den Schwestern in Töß bei Winterthur gehörten Mezzi von Klingenberg, Beli von Liebenberg (1281), Anne und Elsbete Bilgeri (1299), ${ }^{71}$ zu den Schwestern in Otenbach Anna Bilgeri (1335), Amalie von Landenberg (1340), Agnes von Landenberg (1318f.), Gertrud von Landenberg (1318), Bela Schafli (1314), Guta Schafli (1314), Katharina Schafli $(1314)^{72}$ und $\mathrm{zu}$ den Schwestern in Katharinental Luggart von Clingenberg, Sophia von Liebenberg, Guta von Liebenberg, Elsbeth von Liebenberg, Cecilia von Casteln, Agnes von Clingenberg, Clara von Landenberg, Verena von Landenberg und viele Schwestern mit dem Zusatz 'von Schaffusen' und 'von Costentz', bei denen Herkunftsbezeichnung oder Familienzugehörigkeit nicht eindeutig bestimmt werden kann. ${ }^{73}$

Weltliche und geistliche Literatur wurden von den Angehörigen derselben sozialen Schicht gesammelt, produziert und rezipiert. Interferenzen zwischen beiden Bereichen finden darin ihre Erklärung, so $\mathrm{da} \beta$ das Reden über die Gottesminne in der Predigt Rudolfs von Klingenberg fraglos im Rückgriff auf profane Minnedidaxe erfolgt, die den literarisch Gebildeten an die Tageliedsituation erinnert:

' Zitiert nach Frieder Schanze, 'Zur Liederhandschrift $X$ ', in Deutsche Handschriften (Anm. 1), S.316-329, Zitat S.317; Helmut Maurer, Konstanz im Mittelalter. Bd. I: Von den Anfängen bis zum Konzil, Konstanz 1989, S.264.

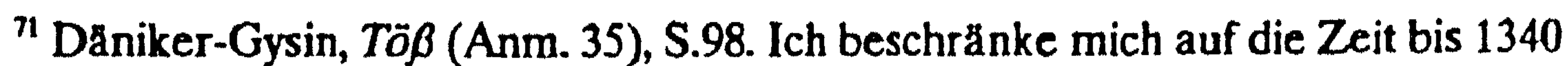
und Familien, deren Namen im Zusammenhang mit Hadlaubs Lied 2 erwähnt worden sind oder aus denen unsere dominikanischen Autoren stammen.

1 Annemarie Halter, Geschichte des Dominikanerinnen-Klosters Oetenbach in Zürich 1234-1525, Phil. Diss. Zürich 1957, S.173-191.

73 Rudolf Henggeler, 'Der Totenrodel des Klosters Katharinenthal bei Dießenhofen', Zeitschrift für Schweizerische Kirchengeschichte 26 (1932), 154-188. Ich beschränke mich hier auf eine repräsentative Auswahl von Namen, da der Katharinentaler Totenrodel keine Jahreszahlen enthält. 
wann als ein geminnetes von seinem geminten schaiden mus vnd es im vrlaub geben wil so erzaiget es sich im sunderlich lieb. vnd trücket es zu im an sein hercz. $\left(92^{\mathrm{vb}}\right)$. 


\section{Korrektur}

Zu Anm. 47:

Dort wird gesagt, daß die genannten Johannes-Libelli keine Parallelüberlieferung zu der vorgestellten Pommersfeldener Handschrift enthalten. Richtig ist, da $B$ die anonyme Predigt auf Johannes Ev. in Pommersfelden, Gräfl. Schönbornsche Bibliothek, cod. 120, fol. 69rb-79ra gekürzt, bearbeitet und im Verbund mit fremdem Predigtmaterial ebenfalls in der Handschrift Karlsruhe, Badische LB, cod. St. Peter pap. 21, fol. $174^{\mathrm{r}}-190^{\mathrm{v}}$ überliefert wird. Textteile derselben Predigt, aber unter anderem Initium werden in Bamberg, SB, cod. hist 153, fol. 220rff. überliefert. Die Predigt Heinrichs von Schaffhausen (Pommersfelden, fol. 79ra $89^{\mathrm{ra}}$ ) findet sich bearbeitet, gekürzt, umgestellt und anonym in der Handschrift Basel, UB, A.VI.38, fol. 182 vb-191 ${ }^{\text {rb }}$ (vgl. dazu Kurt Ruh, in 2VL IV [1983], 1194).

Darüber hinaus liegen anonyme, gekürzte und bearbeitete Parallelüberlieferungen im Kontext des sogenannten Hochalemannischen Predigers in der Handschrift München, BSB, Cgm 531 vor: Die Predigt Konrads von Liebenberg (Pommersfelden, fol. 61 vb-69rb) auf fol. $77^{\mathrm{vb}}-79^{\mathrm{va}}$, die Hugos von Konstanz (Pommersfelden, fol. 79ra-89ra) auf fol. $79^{\mathrm{va}}-80^{\mathrm{vb}}$ und die Heinrichs von Schaffhausen (Pommersfelden, fol. $79^{\mathrm{ra}}-89^{\mathrm{ra}}$ ) auf fol. $100^{\mathrm{ra}}-101^{\mathrm{vb}}$.

Der freundliche Hinweis von Brigitte Degler-Spengler (Basel/Helvetia sacra) auf den neu erschienenen Band 'Das Bistum Konstanz, das Erzbistum Mainz, das Bistum St. Gallen, T. 1-2 (Helvetia sacra Abt. 1, Bd. 2), Basel/Frankfurt a. M. 1993 ' konnte nicht mehr berücksichtigt werden. 